\title{
Intermittent Withdrawal of Oxaliplatin for Alleviating Neurotoxicity during Oxaliplatin-Based Chemotherapy for Japanese Patients with Inoperable or Metastatic Colorectal Cancer: A Phase 2 Multicenter Study
}

\author{
Shunsuke Kato, ${ }^{1, *}$ Hiroo Imai, ${ }^{1, *}$ Makio Gamoh, ${ }^{2}$ Takenori Takahata, ${ }^{3}$ \\ Hisatsugu Ohori, ${ }^{2}$ Katsuhiro Yasuda, ${ }^{4}$ Tomohito Niitani, ${ }^{5}$ Yasuko Murakawa, ${ }^{6}$ \\ Kenji Amagai, ${ }^{7}$ Hideki Isobe, ${ }^{8}$ Yoshiaki Shindo, ${ }^{9}$ Michio Kuroki, ${ }^{10}$ \\ Yasuhiro Sakamoto, ${ }^{2}$ Hideki Shimodaira, ${ }^{1}$ Takashi Yoshioka ${ }^{11}$ and \\ Chikashi Ishioka ${ }^{1}$

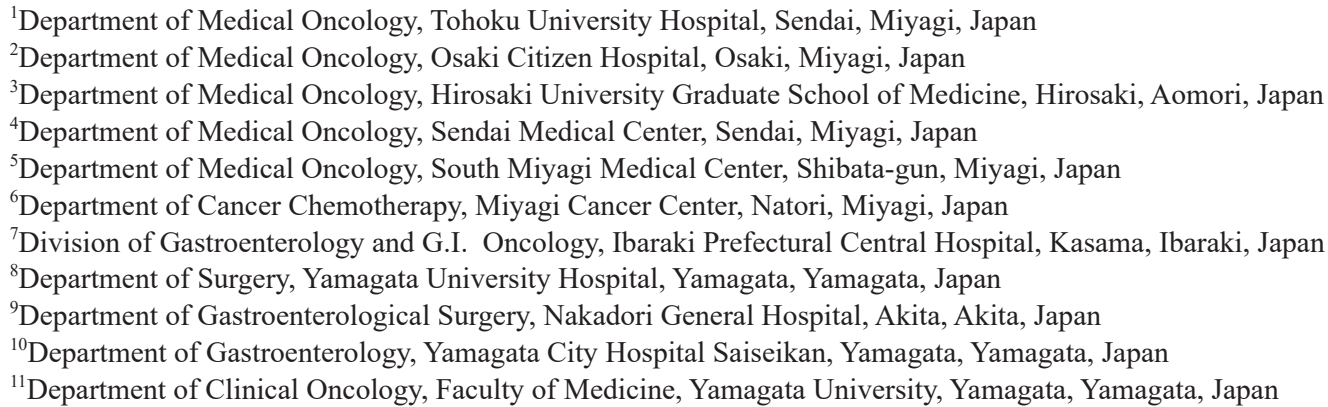

Oxaliplatin-based chemotherapy is a well-established regimen for patients with inoperable and metastatic colorectal cancer. However, one of the major limitations of oxaliplatin-based chemotherapy is sensory neuropathy. It was previously reported that introduction of intermittent oxaliplatin treatment to an oxaliplatin-based regimen has a significant benefit on efficacy or safety. Here, we prospectively assessed whether efficacy and safety of first-line chemotherapy for advanced colorectal cancer are achieved by introduction of withdrawal of oxaliplatin treatment for a certain period (intermittent oxaliplatin treatment). The primary endpoint of the present study is to assess the progression free survival time on patients treated with chemotherapy (mFOLFOX6 (levofolinate, 5-fluorouracil and oxaliplatin combination therapy) plus bevacizumab or CapeOX (oxaliplatin and capecitabine combination therapy) plus bevacizumab) with intermittent oxaliplatin treatment. Bevacizumab is a humanized anti-vascular endothelial growth factor antibody. Median progression-free survival by the mFOLFOX6 plus bevacizumab with intermittent oxaliplatin treatment or the CapeOX plus bevacizumab with intermittent oxaliplatin treatment were 10.6 months $(95 \%$ confidential interval [Cl], 8.3-13.4 months) or 8.0 months $(95 \% \mathrm{Cl}, 4.2-16.8$ months), respectively. Overall response rate by the mFOLFOX6 plus bevacizumab with intermittent oxaliplatin treatment or CapeOX plus bevacizumab with intermittent oxaliplatin treatment was $55.1 \%$ or $42.1 \%$, respectively. Grade 3 or 4 neuropathy was observed in $4.1 \%$ or $10.5 \%$ of patients treated with mFOLFOX6 plus bevacizumab with intermittent oxaliplatin treatment or CapeOX plus bevacizumab with intermittent oxaliplatin treatment, respectively. Introduction of intermittent oxaliplatin treatment has improved severe neuropathy in mFOLFOX6 plus bevacizumab regimen without reducing treatment efficacy.

Keywords: CapeOX plus bevacizumab; colorectal cancer; intermittent oxaliplatin treatment; mFOLFOX6 plus bevacizumab; neuropathy

Tohoku J. Exp. Med., 2018 May, 245 (1), 21-28. (C) 2018 Tohoku University Medical Press

Received March 13, 2018; revised and accepted April 19, 2018. Published online May 9, 2018; doi: 10.1620/tjem.245.21.

*These authors contributed equally to this work.

Correspondence: Chikashi Ishioka, Department of Medical Oncology, Tohoku University Hospital, 4-1 Seiryo-machi, Aoba-ku, Sendai, Miyagi 980-8575, Japan.

e-mail: chikashi@idac.tohoku.ac.jp 


\section{Introduction}

Colorectal cancer is the second and third most common cancer in females and males, respectively, worldwide (Jemal et al. 2011). Surgical resection is the treatment of choice for patients with early-stage colorectal cancer. In patients with resectable metastases, combined resections at the primary and metastatic sites are performed. However, approximately $40 \%$ of patients with colorectal cancer have already progressed to an inoperable stage at the time of diagnosis (Folprecht et al. 2005).

Prior to the 1990s, 5-fluorouracil was the only effective chemotherapeutic agent for inoperable or metastatic colorectal cancer. The introduction of oxaliplatin and irinotecan as a chemotherapeutic agents for colorectal cancer resulted in great improvement in response rates (RR) and overall survival (OS) of patients (de Gramont et al. 2000). Since then, oxaliplatin-based chemotherapy as well as irinotecan-based chemotherapy are well established first- or second-line regimens for patients with inoperable or metastatic colorectal cancer (Goldberg et al. 2004).

One of the major limitations of oxaliplatin-based chemotherapy is sensory neuropathy (de Gramont et al. 2000). Previous randomized phase 3 trials showed that many patients treated with oxaliplatin-based regimen experienced severe neuropathy and the ratio of these patients increased as the cycle of chemotherapy progressed (de Gramont et al. 2000; Giacchetti et al. 2000; Gamelin et al. 2008).

The Combined Oxaliplatin Neurotoxicity Prevention Trial (CONcePT) showed that intermittent oxaliplatin treatment resulted in reduction of severe neuropathy and prolongation of median progression-free survival (PFS) in patients treated with oxaliplatin-based chemotherapy (modified [m] FOLFOX7 regimen) (Hochster et al. 2014). Based on the evidence, the National Comprehensive Cancer Network (NCCN) guidelines recommended the withdrawal of oxaliplatin treatment during oxaliplatin-based chemotherapy for patients with advanced colorectal cancer until their neuropathy is resolved (Benson et al. 2014).

In Japan, the most commonly used regimen as firstline treatment for inoperable or metastatic colorectal cancer is mFOLFOX6 (levofolinate, 5-fluorouracil and oxaliplatin combination therapy) plus bevacizumab regimen or CapeOX (oxaliplatin and capecitabine combination therapy) plus bevacizumab regimen. Bevacizumab is a humanized anti-vascular endothelial growth factor antibody. To the best of our knowledge, however, no studies have assessed the efficacy and safety of intermittent withdrawal of oxaliplatin from mFOLFOX6 plus bevacizumab regimen or CapeOX plus bevacizumab regimen. In this phase 2 study, we assessed the efficacy and safety of intermittent withdrawal of oxaliplatin from mFOLFOX6 plus bevacizumab regimen or CapeOX plus bevacizumab regimen in Japanese patients with advanced colorectal cancer.

\section{Patients and Methods}

Patients

Eligible patients included those aged $\geq 20$ years old, with an Eastern Cooperative Oncology Group (ECOG) performance status of 0 or 1, histopathological diagnosis of colon adenocarcinoma or rectal adenocarcinoma and measurable disease according to the Response Evaluation Criteria in Solid Tumor (RECIST), version 1.0 (Therasse et al. 2000). Patients were expected to survive for at least 12 weeks from the day of enrollment to this study and had the following hematological findings: white blood cells $3,000-12,000 / \mathrm{mm}^{3}$; neutrophils $>1,500 / \mathrm{mm}^{3}$; platelets $>100,000 / \mathrm{mm}^{3}$; bilirubin $<1.5$ times the upper limit of normal levels; AST and ALT $<2.5$ times the upper limit of normal levels; ALP $<2.5$ times the upper limit of normal levels; serum creatinine $<$ limit of normal levels; creatinine clearance $<50 \mathrm{ml} / \mathrm{min}$. All patients provided signed informed consent prior to participation in this study.

Exclusion criteria included prior use of any chemotherapy, immunotherapy or radiation therapy. Patients who had an operation within 4 weeks prior to the day of enrollment to the study were also excluded.

Method

This was an open-label, multicenter, phase 2 study. Patients with inoperable or relapsed colorectal cancer who had never been treated with any anticancer drug were recruited. In eligible patients, mFOLFOX6 plus bevacizumab or CapeOX plus bevacizumab were administered as first-line chemotherapy. Patients received bevacizumab $\left(5 \mathrm{mg} / \mathrm{kg}\right.$ ) followed by mFOLFOX6 (oxaliplatin $85 \mathrm{mg} / \mathrm{m}^{2}$; $l$-leucovorin $200 \mathrm{mg} / \mathrm{m}^{2}$; intravenous bolus of fluorouracil $400 \mathrm{mg} / \mathrm{m}^{2}$, continuous infusion of fluorouracil $2,400 \mathrm{mg} / \mathrm{m}^{2}$ ) or bevacizumab followed by CapeOX (oral administration of capecitabine twice daily on days 1-14 and intravenous administration of oxaliplatin $130 \mathrm{mg} / \mathrm{m}^{2}$ on day 1 of each 21-day cycle). The selection of regimen (mFOLFOX6 or CapeOX) administered to patients depended on the judgment of the treating physician.

After 6 cycles of mFOLFOX6 plus bevacizumab or 4 cycles of CapeOX plus bevacizumab treatment, patients received 6 cycles of sLV5FU2 ( $l$-leucovorin $200 \mathrm{mg} / \mathrm{m}^{2}$; intravenous bolus of fluorouracil $400 \mathrm{mg} / \mathrm{m}^{2}$, continuous infusion of fluorouracil $2,400 \mathrm{mg} / \mathrm{m}^{2}$ ) plus bevacizumab or capecitabine plus bevacizumab (maintenance therapy), with oxaliplatin withdrawal until completion of 6 cycles or 4 cycles of maintenance therapy, respectively (intermittent oxaliplatin treatment). After completion of maintenance therapy, patients received 6 additional cycles of mFOLFOX6 plus bevacizumab or 4 cycles of CapeOX plus bevacizumab.

\section{Statistical analysis}

The treating physician assessed response and time to progression during the study according to RECIST criteria (Therasse et al. 2000) and categorized patients as either responders (those who achieved complete response [CR; all signs of cancer disappearing with treatment] or partial response [PR; defined as a $\geq 30 \%$ reduction in the diameter of measurable lesions on computed tomography [CT]]) or non-responders (those with stable disease [SD; defined as a $<30 \%$ reduction and a $<20 \%$ increase in the diameter of measurable lesions as shown on $\mathrm{CT}]$ ) or progressive disease [PD; defined as a $\geq 20 \%$ increase in the diameter of measurable lesions as shown on $\mathrm{CT}])$. The rate of $\mathrm{CR}$ the PR rate were combined and were used as 
the response rate (RR). The rate of $\mathrm{CR}$, the rate of PR, and the rate of $\mathrm{SD}$ were combined, and these rates were used as the disease control rate (DCR). Statistical analysis of categorical variables was performed using the $\chi^{2}$ test and RR was defined according to RECIST criteria. Median PFS and median time to treatment failure (TTF) were calculated using the Kaplan-Meier method. Differences were identified using Fisher's exact test and were considered significant when $p<0.05$. All statistical analyses were performed using JMP ${ }^{\circledR}$ 11 (SAS Institute Inc., Cary, NC, USA).

Based on data from previous studies (Tournigand et al. 2006, Hochster et al. 2008), the expected median PFS was set at 10 months and the threshold of PFS at 7 months (Saltz et al. 2000) to determine the sample size. According to these settings, it was estimated that a minimum of 58 patients $(\alpha=0.05, \beta=0.2$, Fisher exact test) were required in this study. Assuming a $10 \%$ discontinuation rate from the total patient population, the final sample size was determined as 65 patients.

Primary endpoint of the present study is to assess the progression free survival time of patients with advanced colorectal cancer treated with oxaliplatin based regimen introduced with intermittent oxaliplatin treatment. Secondary endpoint of the present study is to assess the response rate, overall survival time, time to treatment fail- ure and neurotoxicity of patients with advanced colorectal cancer treated with oxaliplatin based regimen introduced with intermittent oxaliplatin treatment.

\section{Assessments}

Safety data were collected through observation of patient health status and weekly blood sample examinations by the treating physician for the first 4 weeks of treatment and repeated after the fifth week at the start of each new cycle of treatment. Adverse events (AEs) were evaluated according to the Common Terminology Criteria for Adverse Events, version 4.0 (Tobinai et al. 1993), and effectiveness was evaluated according to the RECIST criteria (Therasse et al. 2000). Computed tomographic scans were performed every 8 weeks.

\section{Results}

\section{Patient characteristics}

A total of 68 patients were enrolled from 10 institutes of the Tohoku Clinical Oncology Research and Education Society from June 2009 to March 2012. The background characteristics of these patients are listed in Table 1. A total of 49 patients were treated with

Table 1. Patient characteristics.

\begin{tabular}{|c|c|c|}
\hline & $\mathrm{B}+\mathrm{mFOLFOX} 6$ & B + CapeOX \\
\hline Number of patients & 49 & 19 \\
\hline \multicolumn{3}{|l|}{ Sex } \\
\hline male & 33 & 17 \\
\hline female & 16 & 2 \\
\hline Median age (range) & $63(35-81)$ & $68(47-77)$ \\
\hline \multicolumn{3}{|l|}{ Primary site } \\
\hline colon & 29 & 14 \\
\hline rectum & 20 & 5 \\
\hline \multicolumn{3}{|l|}{ Resection of primary lesion } \\
\hline yes & 35 & 14 \\
\hline no & 14 & 5 \\
\hline \multicolumn{3}{|l|}{ Histology } \\
\hline well differentiated & 13 & 5 \\
\hline moderately differentiated & 31 & 11 \\
\hline poorly differentiated & 3 & 2 \\
\hline unknown & 2 & 1 \\
\hline \multicolumn{3}{|l|}{ Performance status } \\
\hline 0 & 40 & 16 \\
\hline 1 & 5 & 1 \\
\hline unknown & 4 & 2 \\
\hline \multicolumn{3}{|l|}{ Adjvant chemotherapy } \\
\hline yes & 13 & 1 \\
\hline no & 36 & 18 \\
\hline \multicolumn{3}{|l|}{ Number of distal metastasis } \\
\hline 1 & 20 & 7 \\
\hline 2 & 16 & 8 \\
\hline$\geqq 3$ & 12 & 1 \\
\hline unknown & 1 & 3 \\
\hline \multicolumn{3}{|l|}{ Post study treatment } \\
\hline CPT-11 based regimen plus Bevacizumab & 20 & 9 \\
\hline CPT-11 plus anti-EGFR antibody & 6 & 2 \\
\hline Capecitabine plus bevacizumab & 18 & 6 \\
\hline Anti-EGFR antobody alone & 5 & 2 \\
\hline Surgical operation & 3 & 1 \\
\hline none & 6 & 2 \\
\hline Relative dose intensity of oxaliplatin (\%) & 70.0 & 71.8 \\
\hline
\end{tabular}

$\mathrm{B}+\mathrm{mFOLFOX6}$, bevacizumab plus mFOLFOX6; $\mathrm{B}+\mathrm{CapeOX}$, bevacizumab plus CapeOX.

CPT-11: Irinotecan. 
mFOLFOX6 plus bevacizumab (mFOLFOX6 plus bevacizumab group) and 19 patients were treated with CapeOX plus bevacizumab (CapeOX plus bevacizumab group). Relative dose intensity of oxaliplatin in mFOLFOX6 plus bevacizumab group and CapeOX plus bevacizumab group in the present study were $70.0 \%$ and $71.8 \%$, respectively.

\section{Efficacy}

Of the 68 enrolled patients, four patients were not assessable for PFS (received resection of measurable lesion). Kaplan-Meier curves of the total population are shown in Fig. 1. Median PFS in the mFOLFOX6 plus bevacizumab group and CapeOX plus bevacizumab groups 11.3 months (95\% CI, 9.1-15.5 months) and 8.0 months
(95\% CI, 4.2-16.8 months), respectively. Kaplan-Meier curves of TTF for mFOLFOX6 plus bevacizumab group and CapeOX plus bevacizumab group are shown in Fig. 2, respectively. The median TTF of mFOLFOX6 plus bevacizumab group and CapeOX plus bevacizumab group were 7.9 months (95\% CI, 6.4-9.1 months) and 5.8 months (95\% CI, 3.1-8.8 months), respectively. Reasons for termination of the oxaliplatin-based regimens in the present study are listed in Table 2.

Kaplan-Meier curves of OS for the mFOLFOX6 plus bevacizumab group and CapeOX plus bevacizumab group are shown in Fig. 3, respectively. The median OS of mFOLFOX6 plus bevacizumab group and CapeOX plus bevacizumab group were 29.0 months (95\% CI, 24.4-40.9

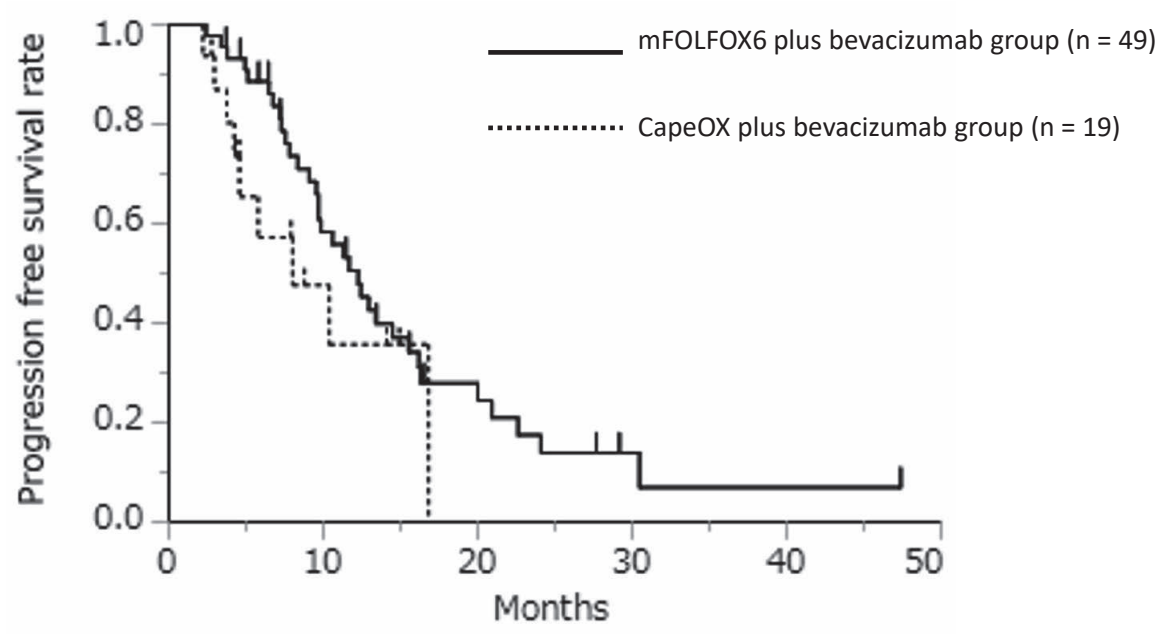

Fig. 1. Kaplan-Meier curve of progression free survival.

Kaplan-Meier curve of progression free survival for mFOLFOX6 plus bevacizumab group (solid line) and CapeOX plus bevacizumab group (dotted line). The vertical axis represents progression free survival rate and the horizontal axis represents progression free survival time.

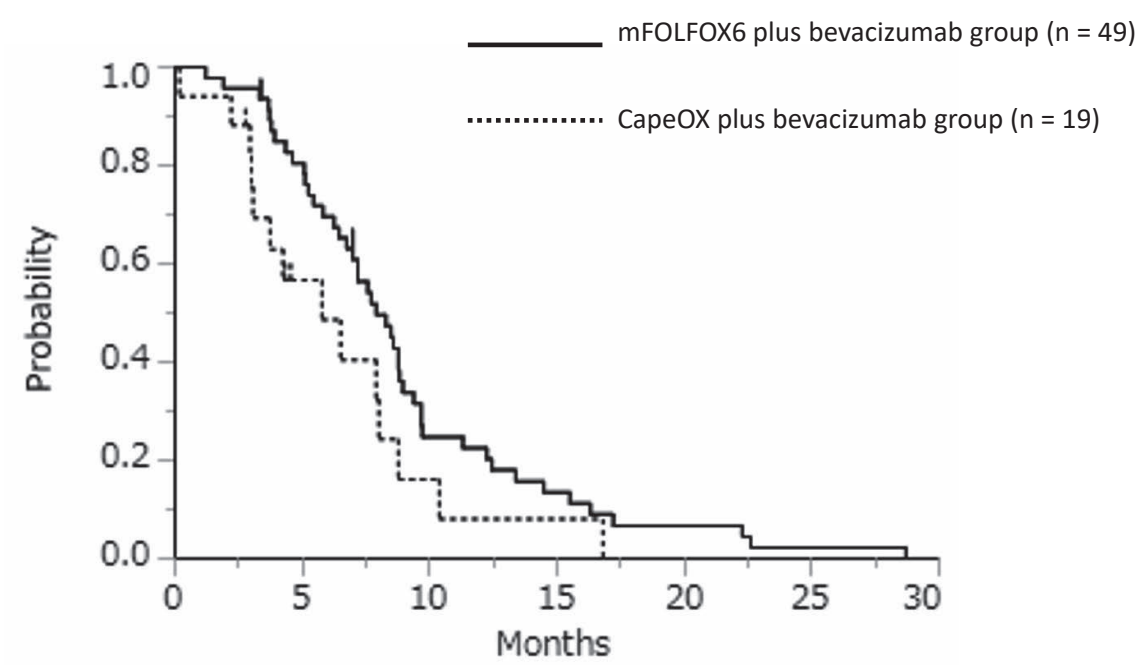

Fig. 2. Kaplan-Meier curve of time to treatment failure.

Kaplan-Meier curve of time to treatment failure for mFOLFOX6 plus bevacizumab group (solid line) and CapeOX plus bevacizumab group (dotted line). The vertical axis represents the rate of patients who could be continued oxaliplatinbased chemotherapy. The horizontal axis represents the time to treatment failure. 
Table 2. Reasons for termination of intermittent oxaliplatin-based chemotherapy in the present study.

\begin{tabular}{lrr}
\hline Reason & B+mFOLFOX6 (\%) & B+CapeOX (\%) \\
\hline Disease progression & $18(37.5)$ & $8(40.0)$ \\
Adverse event & $18(37.5)$ & $5(25.0)$ \\
Patient request & $2(4.2)$ & $1(5.0)$ \\
Resection of distant metastases & $2(4.2)$ & $2(10.0)$ \\
Other reasons & $0(0)$ & $2(10.0)$ \\
Treatment-related death & $1(2.1)$ & $1(5.0)$ \\
Continuation of oxaliplatin-based chemother & $7(14.6)$ & $0(0)$ \\
Unknown & $0(0)$ & $1(5.0)$ \\
\hline
\end{tabular}

$\mathrm{B}+\mathrm{mFOLFOX6}$, bevacizumab plus mFOLFOX6; B+CapeOX, bevacizumab plus CapeOX.

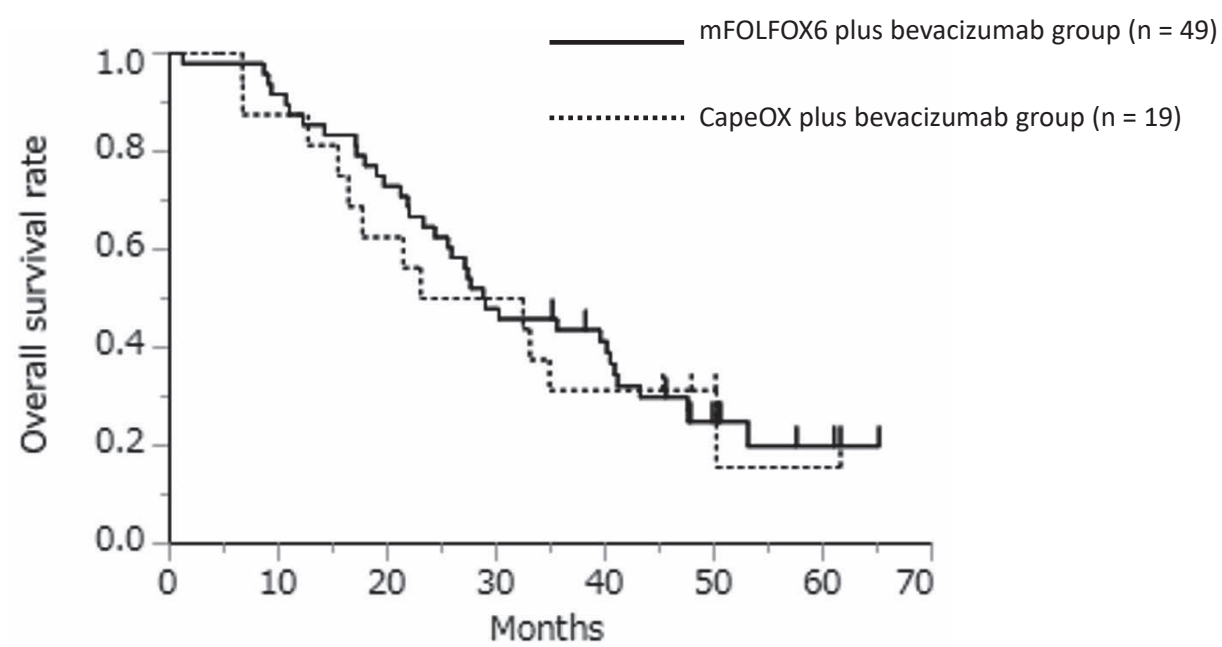

Fig. 3. Kaplan-Meier curve of overall survival.

Kaplan-Meier curve of overall survival for mFOLFOX6 plus bevacizumab group (solid line) and CapeOX plus bevacizumab group (dotted line). The vertical axis represents survival rate and the horizontal axis represents overall survival survival time.

Table 3. Response of intermittent oxaliplatin chemotherapy in advanced colorectal cancers.

\begin{tabular}{rrr}
\hline Best response & B+mFOLFOX6 $(\mathrm{n}=49)$ & B+CapeOX $(\mathrm{n}=19)$ \\
\hline $\mathrm{CR}$ & 2 & 0 \\
$\mathrm{PR}$ & 25 & 8 \\
$\mathrm{SD}$ & 21 & 8 \\
$\mathrm{PD}$ & 0 & 1 \\
$\mathrm{NE}$ & 1 & 2 \\
response rate $(\%)$ & 55.1 & 42.1 \\
Disease control rate $(\%)$ & 98.0 & 84.2 \\
\hline
\end{tabular}

$\mathrm{CR}$, complete response; $\mathrm{PR}$, partial response; $\mathrm{SD}$, stable disease; $\mathrm{PD}$, progression disease; NE, not evaluated; $\mathrm{B}+\mathrm{mFOLFOX6}$, bevacizumab plus mFOLFOX6; B+CapeOX, bevacizumab plus CapeOX.

months) and 22.5 months (95\% CI, 15.5-34.9 months), respectively.

RR and DCR are shown in Table 3. RR of mFOLFOX6 plus bevacizumab or CapeOX plus bevacizumab were $55.1 \%$ or $42.1 \%$, respectively. DCR of mFOLFOX6 plus bevacizumab or CapeOX plus bevacizumab were $98.0 \%$ and $84.2 \%$, respectively.

\section{Toxicity}

The observed toxicity of intermittent oxaliplatin-based chemotherapy is shown in Table 4. The most frequently reported AEs were neutropenia ( $\geq$ grade 3) (28.3\%) followed by hypertension (10.4\%), anorexia $(9.0 \%)$ and handfoot syndrome (7.5\%).

Neuropathy has been reported to be a dose-limiting AE for oxaliplatin-based chemotherapy (de Gramont et al. 
Table 4. Toxicity of intermittent oxaliplatin-based chemotherapy.

\begin{tabular}{rrr}
\hline & \multirow{2}{*}{ Grade3 $(\%)$} & \\
Adverse event & B+mFOLFOX6 & B+CapeOX \\
\hline Leucopenia & $1(2.0)$ & $0(0)$ \\
Neutropenia & $18(36.7)$ & $1(5.3)$ \\
Thrombocytopenia & $1(2.0)$ & $1(5.3)$ \\
Anorexia & $4(8.2)$ & $2(10.5)$ \\
Nausea & $3(6.1)$ & $1(5.3)$ \\
Diarrhea & $1(2.0)$ & $2(10.5)$ \\
Mucositis & $0(0)$ & $1(5.3)$ \\
Bleeding & $0(0)$ & $1(5.3)$ \\
Hypertension & $4(8.2)$ & $3(15.8)$ \\
Proteinuria & $3(6.1)$ & $0(0)$ \\
Hand-foot syndrome & $4(8.2)$ & $1(5.3)$ \\
Anaphylaxis & $2(4.1)$ & $1(5.3)$ \\
Heart failure & $1(2.0)$ & $0(0)$ \\
Cerebral infarction & $1(2.0)$ & $0(0)$ \\
Neuropathy & $2(4.1)$ & $2(10.5)$ \\
\hline B+mFOLFOX6, bevacizumab plus mFOLFOX6; B+CapeOX, bevacizumab plus
\end{tabular}

2000; Giacchetti et al. 2000; Gamelin et al. 2008). In the present study, neuropathy (grade 3 or 4) was observed in $6.0 \%, 4.1 \%$ and $10.5 \%$, of the total population, mFOLFOX6 plus bevacizumab and CapeOX plus bevacizumab groups, respectively.

\section{Discussion}

The aim of this phase 2 study was to assess the efficacy and safety of introduction of intermittent oxaliplatin treatment to the mFOLFOX6 plus bevacizumab or CapeOX plus bevacizumab regimens. Previous evidence (Hochster et al. 2014) demonstrated that the median PFS and median TTF in patients treated with FOLFOX7 plus bevacizumab and intermittent oxaliplatin treatment were 12.0 months and 5.7 months, respectively. These were significantly longer than those of the same regimen without intermittent oxaliplatin treatment (7.4 months and 4.2 months, respectively) (Hochster et al. 2014). Therefore, the effect of intermittent oxaliplatin use on treatment outcome of mFOLFOX6 plus bevacizumab or CapeOX plus bevacizumab regimens was investigated, and the results were compared to those of previous reports.

The median PFS, median OS, median TTF and overall RR in the mFOLFOX6 plus bevacizumab group in the present study were11.3 months, 29.0 months, 7.7 months and $55.1 \%$, respectively. Several previous prospective studies reported that median PFS, OS, TTF and overall RR in patients treated with the mFOLFOX6 plus bevacizumab regimen without intermittent oxaliplatin treatment were 9.015.4 months, 16.5-30.1 months, 5.4 months and 34.762.0\%, respectively (Nishina et al. 2013; O’Neil et al. 2014; Van Cutsem et al. 2015; Yamazaki et al. 2016). The efficacy of the mFOLFOX6 plus bevacizumab regimen with intermittent oxaliplatin treatment in the present study was approximately equivalent to those, suggesting similar efficacy with or without intermittent oxaliplatin treatment in patients with advanced and metastatic colorectal cancer. In the present study, the median PFS, median OS, median TTF and overall RR in the CapeOX plus bevacizumab group were 8.0 months, 22.5 months, 5.8 months and $42.1 \%$, respectively. Several previous prospective studies reported that median PFS, median OS, and overall RR of the CapeOX plus bevacizumab regimen without intermittent oxaliplatin treatment were 9.6-10.0 months, 23.2-34.6 months, 6.7 days and 52.2-59.3\%, respectively (Hurwitz et al. 2012; Yalcin et al. 2013; Uchima et al. 2014; Benson et al. 2016; Ogata et al. 2016). The median PFS and median OS, median TTF and overall RR by CapeOX plus bevacizumab regimen with intermittent oxaliplatin treatment in the present study was slightly worse than those of the CapeOX plus bevacizumab regimen without intermittent oxaliplatin treatment in previous reports. These results suggests that introduction of intermittent oxalipatin treatment resulted in comparable effects only in mFOLFOX6 plus bevacizumab group but not in $\mathrm{CapeOX}$ plus bevacizumab group in the present study.

Subsequently, the rate of neuropathy (grade 3 or 4) incidence, a major dose-limiting $\mathrm{AE}$ for oxaliplatin treatment (de Gramont et al. 2000) was compared between the present and previous studies. In the present study, the rate of neuropathy in the mFOLFOX6 plus bevacizumab group was $4.1 \%$, whereas that of patients treated with the same regimen without intermittent oxaliplatin treatment in previous prospective studies was 7.3-22.0\% (Nishina et al. 2013; O'Neil et al. 2014; Van Cutsem et al. 2015; Yamazaki et al. 2016). In the CapeOX plus bevacizumab group, the rate of neuropathy was $10.5 \%$, whereas that of patients treated with the same regimen without intermittent oxaliplatin treatment in previous studies (three prospective and one retrospective) were 0-15.0\% (Hurwitz et al. 2012; Yalcin et al. 2013; Uchima et al. 2014; Ogata et al. 2016). Based on these data, intermittent oxaliplatin treatment ameliorates the 
incidence of neuropathy grade when administered together with the mFOLFOX6 plus bevacizumab regimen.

A limitation of this study is the small sample size, especially the 18 patients in the CapeOX plus bevacizumab group. Moreover, this was an uncontrolled study, with all patients receiving intermittent oxaliplatin treatment and oxaliplatin based regimen treated in the present study (mFOLFOX6 or CapeOX) was physician's choice. Because statistical power in the present study design was weak, we could not reveal the reason why the efficacies and toxicities by introduction of intermittent oxaliplatin treatment were ameliorated only in mFOLFOX6 plus bevacizumab group but not in CapeOX plus bevacizumab group.

As long as we compare the treatment result of introduction of intermittent oxaliplatin treatment to mFOLFOX6 or CapeOX plus bevacizuman regimen in the present study to those without intermittent oxaliplatin treatment in previous reports, this phase 2 study suggests that introduction of intermittent oxaliplatin treatment to mFOLFOX6 plus bevacizumab regimen often used to Japanese patients is useful treatment option to reduce the incident rate of neurotoxicity and to maintain the treatment effect of oxaliplatin based chemotherapy. Phase 3 study to investigate the potency of introduction of intermittent oxaliplatin treatment in mFOLFOX6 plus bevacizumab regimen is warranted.

\section{Acknowledgments}

This study was supported by the Tohoku Clinical Oncology Research and Education Society (T-CORE 0901 trial, UMIN 000002042).

\section{Conflict of Interest}

The Tohoku Clinical Oncology Research and Education was the sponsor of this trial.

Chikashi Ishioka has received research funding from the Tokyo Cooperative Oncology Group. Chikashi Ishioka has also received contributions from Chugai Pharmaceutical, Ono pharmaceutical, MSD, Phizer, AstraZeneca, Bristol-Myers squibb, Janssen Pharmaceutical, Taiho Pharmaceutical, Daiichi Sankyo Company, Limited, and Takeda Pharmaceutical. Chikashi Ishioka is a representative of Tohoku Clinical Oncology Research and Education Society, a specified nonprofit corporation.

Hideki Shimodaira has received contributions from Taiho, Eizai and Bayer.

Shunske Kato has received research funding from Ono pharmatheutical.

However, all authors declare no conflict of interest concerning the present study.

\section{References}

Benson, A.B. 3rd, Kiss, I., Bridgewater, J., Eskens, F.A., Sasse, C., Vossen, S., Chen, J., Van Sant, C., Ball, H.A., Keating, A. \& Krivoshik, A. (2016) BATON-CRC: a phase II randomized trial comparing tivozanib plus mFOLFOX6 with bevacizumab plus mFOLFOX6 in stage IV metastatic colorectal cancer. Clin. Cancer Res., 22, 5058-5067.

Benson, A.B., 3rd, Venook, A.P., Bekaii-Saab, T., Chan, E., Chen, Y.J., Cooper, H.S., Engstrom, P.F., Enzinger, P.C., Fenton, M.J., Fuchs, C.S., Grem, J.L., Hunt, S., Kamel, A., Leong, L.A., Lin, E., et al. (2014) Colon cancer, version 3.2014. J.
Natl. Compr. Canc. Netw.,12, 1028-1059.

de Gramont, A., Figer, A., Seymour, M., Homerin, M., Hmissi, A., Cassidy, J., Boni, C., Cortes-Funes, H., Cervantes, A., Freyer, G., Papamichael, D., Le Bail, N., Louvet, C., Hendler, D., de Braud, F., et al. (2000) Leucovorin and fluorouracil with or without oxaliplatin as first-line treatment in advanced colorectal cancer. J. Clin. Oncol., 18, 2938-2947.

Folprecht, G., Grothey, A., Alberts, S., Raab, H.R. \& Kohne, C.H. (2005) Neoadjuvant treatment of unresectable colorectal liver metastases: correlation between tumour response and resection rates. Ann. Oncol., 16, 1311-1319.

Gamelin, L., Boisdron-Celle, M., Morel, A., Poirier, A.L., Berger, V., Gamelin, E., Tournigand, C. \& de Gramont, A. (2008) Oxaliplatin-related neurotoxicity: interest of calcium-magnesium infusion and no impact on its efficacy. J. Clin. Oncol., 26, 1188-1189; author reply 1189-1190.

Giacchetti, S., Perpoint, B., Zidani, R., Le Bail, N., Faggiuolo, R., Focan, C., Chollet, P., Llory, J.F., Letourneau, Y., Coudert, B., Bertheaut-Cvitkovic, F., Larregain-Fournier, D., Le Rol, A., Walter, S., Adam, R., et al. (2000) Phase III multicenter randomized trial of oxaliplatin added to chronomodulated fluorouracil-leucovorin as first-line treatment of metastatic colorectal cancer. J. Clin. Oncol., 18, 136-147.

Goldberg, R.M., Sargent, D.J., Morton, R.F., Fuchs, C.S., Ramanathan, R.K., Williamson, S.K., Findlay, B.P., Pitot, H.C. \& Alberts, S.R. (2004) A randomized controlled trial of fluorouracil plus leucovorin, irinotecan, and oxaliplatin combinations in patients with previously untreated metastatic colorectal cancer. J. Clin. Oncol., 22, 23-30.

Hochster, H.S., Grothey, A., Hart, L., Rowland, K., Ansari, R., Alberts, S., Chowhan, N., Ramanathan, R.K., Keaton, M., Hainsworth, J.D. \& Childs, B.H. (2014) Improved time to treatment failure with an intermittent oxaliplatin strategy: results of CONcePT. Ann. Oncol., 25, 1172-1178.

Hochster, H.S., Hart, L.L., Ramanathan, R.K., Childs, B.H., Hainsworth, J.D., Cohn, A.L., Wong, L., Fehrenbacher, L., Abubakr, Y., Saif, M.W., Schwartzberg, L. \& Hedrick, E. (2008) Safety and efficacy of oxaliplatin and fluoropyrimidine regimens with or without bevacizumab as first-line treatment of metastatic colorectal cancer: results of the TREE Study. $J$. Clin. Oncol., 26, 3523-3529.

Hurwitz, H., Mitchell, E.P., Cartwright, T., Kwok, A., Hu, S., McKenna, E. \& Patt, Y.Z. (2012) A randomized, phase II trial of standard triweekly compared with dose-dense biweekly capecitabine plus oxaliplatin plus bevacizumab as first-line treatment for metastatic colorectal cancer: XELOX-A-DVS (dense versus standard). Oncologist, 17, 937-946.

Jemal, A., Bray, F., Center, M.M., Ferlay, J., Ward, E. \& Forman, D. (2011) Global cancer statistics. CA Cancer J. Clin., 61, 69-90.

Nishina, T., Takano, Y., Denda, T., Yasui, H., Takeda, K., Ura, T., Esaki, T., Okuyama, Y., Kondo, K., Takahashi, Y., Sugiyama, Y. \& Muro, K. (2013) A phase II clinical study of mFOLFOX6 plus bevacizumab as first-line therapy for Japanese advanced/ recurrent colorectal cancer patients. Jpn. J. Clin. Oncol., 43, 1080-1086

O’Neil, B.H., Cainap, C., Van Cutsem, E., Gorbunova, V., Karapetis, C.S., Berlin, J., Goldberg, R.M., Qin, Q., Qian, J., Ricker, J.L., Fischer, J., McKee, M.D., Carlson, D.M. \& Kim, T.W. (2014) Randomized phase II open-label study of mFOLFOX6 in combination with linifanib or bevacizumab for metastatic colorectal cancer. Clin. Colorectal Cancer, 13, 156-163.e152.

Ogata, Y., Shimokawa, M., Tanaka, T., Emi, Y., Oki, E., Saeki, H., Sadanaga, N., Kusumoto, T., Touyama, T., Kimura, M., Baba, H., Akagi, Y., Shirouzu, K. \& Maehara, Y. (2016) A prospective study of XELOX plus bevacizumab as first-line therapy in Japanese patients with metastatic colorectal cancer (KSCC 0902). Int. J. Clin. Oncol., 21, 335-343. 
Saltz, L.B., Cox, J.V., Blanke, C., Rosen, L.S., Fehrenbacher, L., Moore, M.J., Maroun, J.A., Ackland, S.P., Locker, P.K., Pirotta, N., Elfring, G.L. \& Miller, L.L. (2000) Irinotecan plus fluorouracil and leucovorin for metastatic colorectal cancer. $N$. Engl. J. Med., 343, 905-914.

Therasse, P., Arbuck, S.G., Eisenhauer, E.A., Wanders, J., Kaplan, R.S., Rubinstein, L., Verweij, J., Van Glabbeke, M., van Oosterom, A.T., Christian, M.C. \& Gwyther, S.G. (2000) New guidelines to evaluate the response to treatment in solid tumors. J. Natl. Cancer Inst., 92, 205-216.

Tobinai, K., Kohno, A., Shimada, Y., Watanabe, T., Tamura, T., Takeyama, K., Narabayashi, M., Fukutomi, T., Kondo, H., Shimoyama, M., et al. (1993) Toxicity grading criteria of the Japan Clinical Oncology Group. Jpn. J. Clin. Oncol., 23, 250-257.

Tournigand, C., Cervantes, A., Figer, A., Lledo, G., Flesch, M., Buyse, M., Mineur, L., Carola, E., Etienne, P.L., Rivera, F., Chirivella, I., Perez-Staub, N., Louvet, C., Andre, T., TabahFisch, I. \& de Gramont, A. (2006) OPTIMOX1: a randomized study of FOLFOX4 or FOLFOX7 with oxaliplatin in a stopand-go fashion in advanced colorectal cancer: a GERCOR study. J. Clin. Oncol., 24, 394-400.

Uchima, Y., Nishii, T., Iseki, Y., Ishii, M., Hiramatsu, S., Iwauchi, T., Morimoto, J., Kosaka, K., Tei, S. \& Takeuchi, K. (2014) Retrospective analysis of capecitabine and oxaliplatin (XELOX) plus bevacizumab as a first-line treatment for Japanese patients with metastatic colorectal cancer. Mol. Clin. Oncol., 2, 134-138.

Van Cutsem, E., Prenen, H., D’Haens, G., Bennouna, J., Carrato, A., Ducreux, M., Bouche, O., Sobrero, A., Latini, L., Staines, H., Oum'Hamed, Z., Dressler, H., Studeny, M. \& Capdevila, J. (2015) A phase I/II, open-label, randomised study of nintedanib plus mFOLFOX6 versus bevacizumab plus mFOLFOX6 in first-line metastatic colorectal cancer patients. Ann. Oncol., 26, 2085-2091.

Yalcin, S., Uslu, R., Dane, F., Yilmaz, U., Zengin, N., Buyukunal, E., Buyukberber, S., Camci, C., Sencan, O., Kilickap, S., Ozdener, F. \& Cevik, D. (2013) Bevacizumab + capecitabine as maintenance therapy after initial bevacizumab + XELOX treatment in previously untreated patients with metastatic colorectal cancer: phase III 'Stop and Go' study results: a Turkish Oncology Group Trial. Oncology, 85, 328-335.

Yamazaki, K., Nagase, M., Tamagawa, H., Ueda, S., Tamura, T., Murata, K., Eguchi Nakajima, T., Baba, E., Tsuda, M., Moriwaki, T., Esaki, T., Tsuji, Y., Muro, K., Taira, K., Denda, T., et al. (2016) Randomized phase III study of bevacizumab plus FOLFIRI and bevacizumab plus mFOLFOX6 as first-line treatment for patients with metastatic colorectal cancer (WJOG4407G). Ann. Oncol., 27, 1539-1546. 